\title{
TUBE TO ANNULUS-AN EXACT NONLINEAR MEMBRANE SOLUTION*
}

\author{
BY \\ CHIEN-HENG WU \\ University of Illinois at Chicago Circle
}

1. Introduction. The theory of membranes for highly elastic materials has been advanced in the last two decades by Mooney [1], Rivlin [2], Green and Shield [3], Rivlin and Thomas [4], Adkins and Rivlin [5] and other researchers. A comprehensive presentation of this theory can be found in Green and Zerna [6] and Green and Adkins [7].

By using this theory the class of axially symmetrical problems can usually be solved by numerical integration. A different integration procedure has been used by Yang [8] in studying the stress concentration in a rubber sheet. Foster [8] has developed an approximate theory for axially symmetrical membranes based on the assumption that the meridional deformations are large. Very few exact solutions are available.

In this paper we study the problem of stretching a tube into an annulus (Fig. 1). The procedure used is a semi-inverse method in the sense that we specify the inner radius of the annulus in equilibrium and look for the stress required on the outer boundary. The inner boundary of the annulus is assumed to be free of stresses. Exact solutions are obtained for both Mooney material and neo-Hookean material.

2. Formulation. Consider a thin cylindrical tube of radius $R_{0}$ and thickness $h_{0}$.
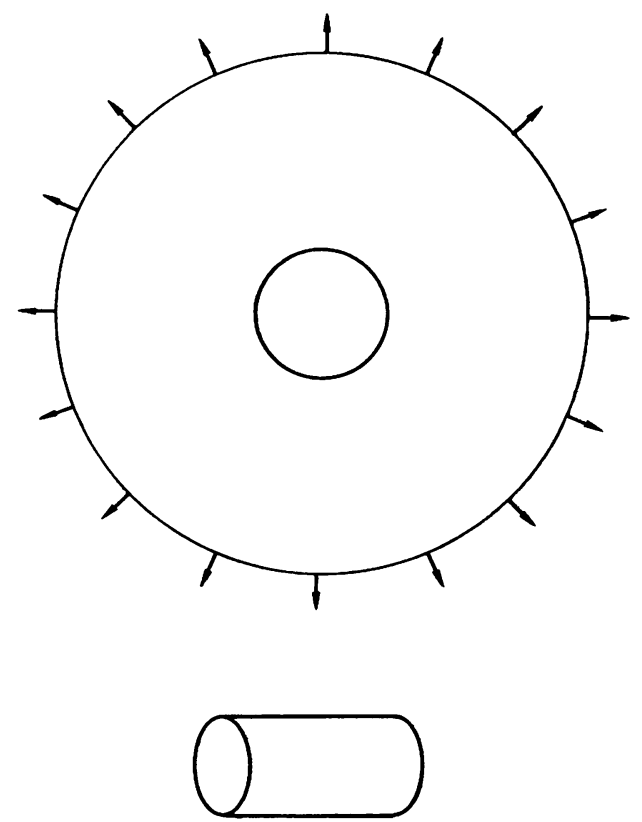

Fig. 1. Tube to annulus.

* Received August 28, 1968. 
The tube is subjected to a very large outward radial extension applied to one of its ends so that the equilibrium configuration is a circular annulus of inner radius $A \geq R_{0}$. The solution of this problem with $A$ as a parameter is studied.

We choose a cylindrical polar coordinate system $\left(\rho, \theta, x_{3}\right)$, the $x_{3}$-axis of which coincides with the axis of symmetry of the tube. We suppose that any point $P_{T}\left(R_{0}, \theta, x_{3}\right)$ in the middle surface $S_{T}$ of the tube is carried by the deformation to the point $P_{A}(R, \theta, 0)$ in the deformed middle surface $S_{A}$ of the annulus. From the symmetry of the problem it follows that principal extension ratios in the radial and circumferential directions are, respectively,

$$
\begin{aligned}
\lambda_{1} & =d R / d x_{3}, \\
\lambda_{2} & =R / R_{0} .
\end{aligned}
$$

If the tube is made of incompressible material, then the principal extension ratio in the direction normal to $S_{A}$ is

$$
\lambda_{3}=1 /\left(\frac{R}{R_{0}} \frac{d R}{d x_{3}}\right) .
$$

The corresponding invariants are

$$
\begin{aligned}
& I_{1}=\lambda_{1}^{2}+\lambda_{2}^{2}+\lambda_{3}^{2}, \\
& I_{2}=\lambda_{1}^{-2}+\lambda_{2}^{-2}+\lambda_{3}^{-2}, \\
& I_{3}=1 .
\end{aligned}
$$
form $^{1}$

When the material is isotropic, and the strain energy function $W$ assumes the linear

$$
W=C\left[\left(I_{1}-3\right)+k\left(I_{2}-3\right)\right]
$$

suggested by Mooney [1], the nonzero physical stress resultants in the radial and circumferential directions are, respectively,

$$
\begin{aligned}
T_{1} & =2 h_{0} \lambda_{3}\left(\lambda_{1}^{2}-\lambda_{3}^{2}\right)\left(\frac{\partial W}{\partial I_{1}}+\lambda_{2}^{2} \frac{\partial W}{\partial I_{2}}\right) \\
& =2 h_{0} \lambda_{3} C\left(\lambda_{1}^{2}-\lambda_{3}^{2}\right)\left(1+k \lambda_{2}^{2}\right), \\
T_{2} & =2 h_{0} \lambda_{3}\left(\lambda_{2}^{2}-\lambda_{3}^{2}\right)\left(\frac{\partial W}{\partial I_{1}}+\lambda_{1}^{2} \frac{\partial W}{\partial I_{2}}\right) \\
& =2 h_{0} \lambda_{3} C\left(\lambda_{2}^{2}-\lambda_{3}^{2}\right)\left(1+k \lambda_{1}^{2}\right) .
\end{aligned}
$$

For the problem under consideration, the equations of equilibrium reduce to the single equation

$$
d\left(R T_{1}\right) / d R=T_{2} .
$$

The boundary conditions are

$$
T_{1}=0, \quad R=A \quad \text { at } \quad x_{3}=0 .
$$

${ }^{1} C$ and $k$ are material constants. The constant $C$ has the dimension of force per unit area and $k$ is dimensionless. 
It is now convenient to introduce the dimensionless variables $z, r(z), t_{1}(z), t_{2}(z)$ and the dimensionless parameter $a$, defined by

$$
\begin{aligned}
z & =x_{3} / R_{0}, \quad r(z)=R\left(x_{3}\right) / R_{0}, \\
t_{1}(z) & =T_{1}\left(x_{3}\right) / 2 h_{0} C, \quad t_{2}(z)=T_{2}\left(x_{3}\right) / 2 h_{0} C, \\
a & =A / R_{0} \quad(a \geq 1) .
\end{aligned}
$$

Upon introducing these quantities into Eqs. (2.1), (2.2), (2.5)-(2.7) we obtain

$$
\begin{gathered}
\lambda_{1}=\frac{d r}{d z}, \quad \lambda_{2}=r, \quad \lambda_{3}=1 / r \frac{d r}{d z}, \\
t_{1}=\left[\frac{1}{r} \frac{d r}{d z}-\left(r \frac{d r}{d z}\right)^{-3}\right]\left(1+k r^{2}\right), \\
t_{2}=\left[\left(\frac{1}{r} \frac{d r}{d z}\right)^{-1}-\left(r \frac{d r}{d z}\right)^{-3}\right]\left[1+k\left(\frac{d r}{d z}\right)^{2}\right], \\
\frac{d}{d r}\left(r t_{1}\right)=t_{2}, \\
t_{1}(0)=0, \quad r(0)=a .
\end{gathered}
$$

Substituting (2.10), (2.11) into (2.12) we obtain a single second-order nonlinear ordinary differential equation for $r$ :

$$
\left\{\left[\frac{1}{r}+\frac{3}{r^{3}\left(\frac{d r}{d z}\right)^{4}}\right]+k\left[r+\frac{3}{r\left(\frac{d r}{d z}\right)^{4}}\right]\right\} \frac{d^{2} r}{d z^{2}}+\frac{3}{r^{4}\left(\frac{d r}{d z}\right)^{2}}-1+k\left[\left(\frac{d r}{d z}\right)^{2}+\frac{1}{r^{4}}\right]=0 .
$$

Our aim is to solve (2.14) with the boundary conditions (2.13).

3. Solution. Eq. (2.14) is highly nonlinear. This equation, however, can be integrated with a simple transformation. We introduce a new function $p(r)$ defined by

$$
p(r)=d r / d z, \quad r \geq a \geq 1 .
$$

It follows from (2.13) and (2.10) that

$$
p(a)=a^{-1 / 2} .
$$

Introducing (3.1) into (2.14) and noting that

$$
d^{2} r / d z^{2}=d p / d z=p d p / d r
$$

we obtain

$$
\frac{d p}{d r}=\frac{p\left[\left(r^{4} p^{2}-3\right)-k\left(r^{4} p^{4}+p^{2}\right)\right]}{r\left[\left(p^{4} r^{2}+3\right)+k\left(r^{4} p^{4}+3 r^{2}\right)\right]}
$$

Eqs. (3.3) and (3.2) constitute a problem for the determination of $p(r)$ for $r \geq a$. Once $p(r)$ is found, Eq. (3.1) can be integrated.

Eq. (3.3) can be easily cast into the form

$$
r^{3} p^{3}\left(p \frac{d p}{d r}-r\right)+3\left(r \frac{d p}{d r}+p\right)+k\left[r^{4} p^{4}\left(r \frac{d p}{d r}+p\right)+r^{3} p^{3}\left(3 p^{-3} \frac{d p}{d r}+\frac{1}{r^{3}}\right)\right]=0 \text {. }
$$


It follows that

$$
\frac{d}{d r}\left[p^{2}-3(r p)^{-2}+k(r p)^{2}-3 k p^{-2}\right]=2\left(r-\frac{k}{r^{3}}\right) .
$$

Integrating (3.4) and applying (3.2), we obtain

$$
p^{2}-3(r p)^{-2}+k(r p)^{2}-3 k p^{-2}=\left(r^{2}-a^{2}\right)-k\left(\frac{1}{a^{2}}-\frac{1}{r^{2}}\right)-\frac{2}{a}-2 k a
$$

and hence

$$
\begin{aligned}
p^{2}(r)= & 2^{-1}\left(1+k r^{2}\right)^{-1}\left[\left(r^{2}-a^{2}-2 a^{-1}\right)-k\left(a^{-2}-r^{-2}+2 a\right)\right. \\
& \left. \pm\left\{\left[\left(r^{2}-a^{2}-2 a^{-1}\right)-k\left(a^{-2}-r^{-2}+2 a\right)\right]^{2}+12 r^{-2}\left(1+k r^{2}\right)^{2}\right\}^{1 / 2}\right] .
\end{aligned}
$$

Since $p^{2}$ is real the minus sign in (3.5) has to be excluded. Moreover, for the problem under consideration, $p(r)$ is positive. Thus

$$
\begin{aligned}
p(r)= & 2^{-1 / 2}\left(1+k r^{2}\right)^{-1 / 2}\left[\left(r^{2}-a^{2}-2 a^{-1}\right)-k\left(a^{-2}-r^{-2}+2 a\right)\right. \\
& \left.+\left\{\left[\left(r^{2}-a^{2}-2 a^{-1}\right)-k\left(a^{-2}-r^{-2}+2 a\right)\right]^{2}+12 r^{-2}\left(1+k r^{2}\right)^{2}\right\}^{1 / 2}\right]^{1 / 2} .
\end{aligned}
$$

For neo-Hookean material $(k=0)$, Eq. (3.5) reduces to the simpler form

$$
p(r)=2^{-1 / 2}\left\{\left(r^{2}-a^{2}-2 a^{-1}\right)+\left[\left(r^{2}-a^{2}-2 a^{-1}\right)^{2}+12 r^{-2}\right]^{1 / 2}\right\}^{1 / 2} .
$$

Equation (3.1) together with (2.13) and (3.6 or 3.7) can now be integrated to yield

$$
z=\int_{a}^{r} p^{-1}(x) d x .
$$

Eq. (3.8) and (3.6 or 3.7) constitute the complete solution.

4. Asymptotic behavior of the solution. The solution obtained in Sec. 3 possesses certain asymptotes as $r$ approaches infinity. Moreover, it appears that the solution approaches the asymptotes very rapidly. We shall investigate these properties for the neoHookean material and the Mooney material separately.

Neo-Hookean material $(k=0)$. It can be seen from (3.7) that

$$
\lim _{r \rightarrow \infty} p(r)=\frac{d r}{d z} \rightarrow r
$$

It follows from (4.9) and (2.9-3.11) that

$$
\begin{aligned}
\lambda_{1} & \rightarrow \lambda_{2}=r, & \lambda_{3} & \rightarrow r^{-2}, \\
t_{1} & \rightarrow 1, & t_{2} & \rightarrow 1 .
\end{aligned}
$$

We note that $\lambda_{3} h_{0}$ is the thickness of the annulus. Eq. (4.1) implies that

$$
r \rightarrow N e^{2}
$$

where $N$ is a constant.

Mooney material $(k \neq 0)$. Eq. (3.6) implies that

$$
\lim _{r \rightarrow \infty} p(r)=\frac{d r}{d z} \rightarrow k^{-1 / 2} \text {. }
$$


It follows from (4.5) and (2.9)-(2.11) that

$$
\begin{array}{rlrl}
\lambda_{1} & \rightarrow k^{-1 / 2}, & \lambda_{3} & \rightarrow k^{1 / 2} r^{-1}, \\
t_{1} & \rightarrow k^{1 / 2} r, & t_{2} \rightarrow 2 k^{1 / 2} r .
\end{array}
$$

Eqs. (4.4), (4.5) also imply that

$$
\begin{gathered}
r \rightarrow k^{-1 / 2} z+M \\
t_{1} \rightarrow z+k^{1 / 2} M \\
t_{2} \rightarrow 2 z+2 k^{1 / 2} M
\end{gathered}
$$

where $M$ is a constant.

5. Results. Two sets of numerical solutions are presented in this section, one for neo-Hookean material and one for Mooney material with $k=0.2$. Both solutions are obtained for three different values of $a$, namely $a=1,2$ and 3 .

For neo-Hookean material, the principal strains are plotted against $z$ in Fig. 2 . It is seen that $\lambda_{1} \rightarrow \lambda_{2}=r$ very rapidly for all values of $a$. The two principal strains $\lambda_{1}$ and $\lambda_{2}$ are practically equal for $z>2$.

It is interesting to note that $\lambda_{2}=r$ is a monotonically increasing function of $z$ for

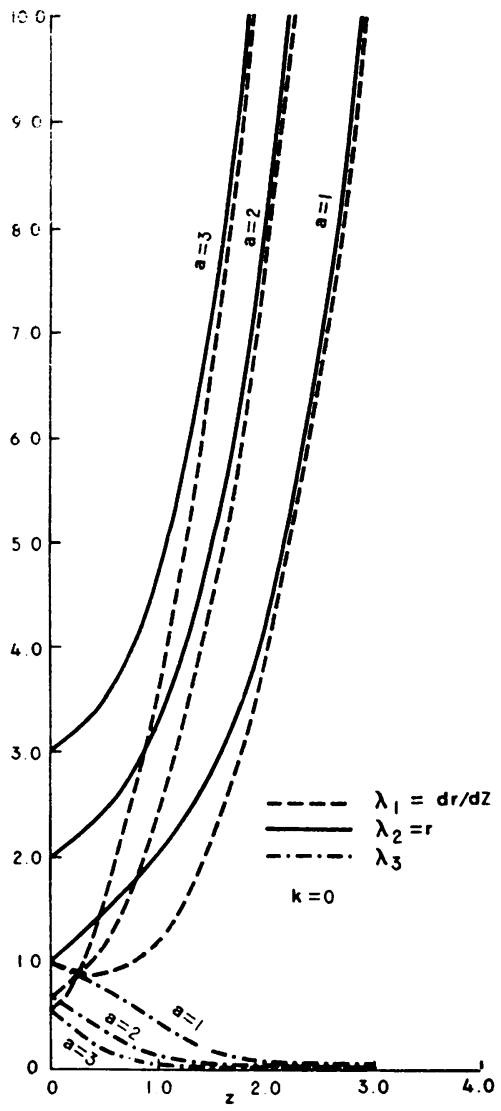

Fig. 2. Principal extension ratios for a neo-Hookean tube. 
all values of $a$ and $\lambda_{1}=d r / d z$ has a minimum for a less than certain value. This value of $a$ can be determined. Equation (3.3) implies that, for $k=0$,

$$
d \lambda_{1} / d z=\lambda_{1} d \lambda_{1} / d r=0 \text { at } r=a
$$

if

$$
a^{4} p^{2}(a)-3=0 .
$$

Substituting (3.2) into (5.1) we find

$$
a=3^{1 / 3}=1.44 .
$$

Thus, for $a \geq 1.44, \lambda_{1}=d r / d z$ is also a monotonically increasing function of $z$.

The radial and circumferential stress resultants are given in Fig. 3. Both approach the asymptotes (4.2) very rapidly. While the radial stress resultant $t_{1}$ is a monotonically increasing function of $z$, the circumferential stress resultant $t_{2}$ has a maximum for a less than certain value. To find this limiting value, we differentiate (2.11) with respect to $r$ to obtain

$$
\frac{d t_{2}}{d z}=p \frac{d t_{2}}{d r}=\frac{r^{4} p^{3}-r^{5} p^{2}(d p / d r)+3(d / d r)(r p)}{r^{4} p^{3}} .
$$

It follows from (5.3) and the condition $t_{2}^{\prime}(0)=0$ that

$$
\left(a^{3}+3\right)-\frac{1}{4}\left(a^{3}-3\right)^{2}=0
$$

and hence $a=2.175$. For $a \geq 2.175, t_{2}$ is a monotonically decreasing function of $z$.

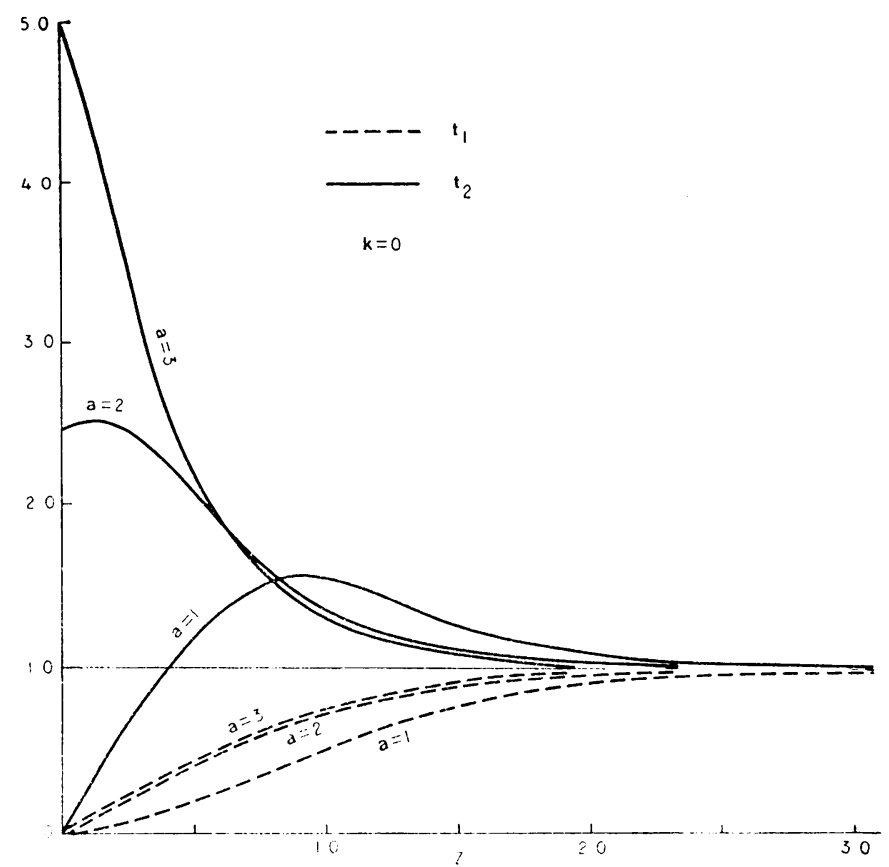

FIg. 3. Stress resultants for a neo-Hookean tube. 


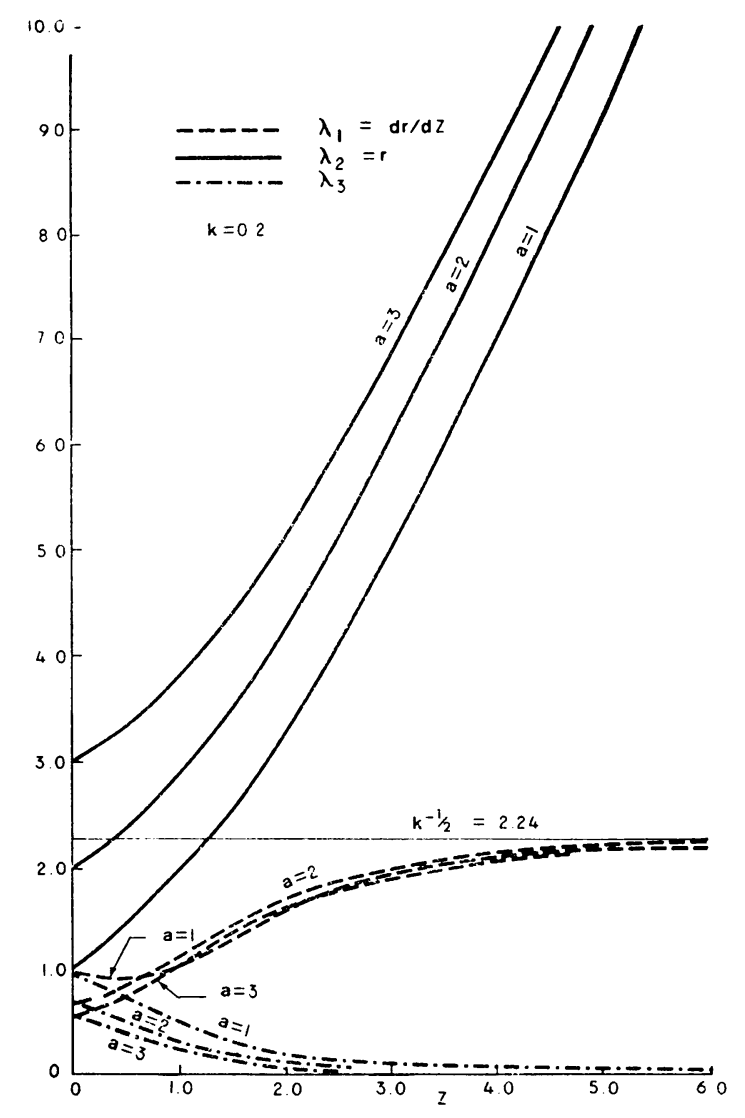

Fig. 4. Principal extension ratios for a Mooney tube.

The principal strains for the case of Mooney material with $k=0.2$ are plotted against $z$ in Fig. 4. The radial and circumferential stress resultants are plotted against $r$ in Fig. 5. The asymptotes (4.5) are also shown in these figures.

For this case, $\lambda_{2}=r$ is a monotonically increasing function of $z$ and $\lambda_{1}=d r / d z$ has a minimum for a less than certain value. This value is again determined from the condition

$$
d \lambda_{1} / d z=p d p / d r=0 \text { at } \quad r=a .
$$

Using (3.2), (3.3) and (5.5) we find

$$
a\left(a^{3}-3\right)-k\left(a^{3}+1\right)=0 .
$$

For $h=0.2$, the root of (5.6) is $a=1.491$. Thus for $k=0.2$ and $a \geq 1.491$, the radial extension ratio $\lambda_{1}$ is a monotonically increasing function of $z$.

Fig. 5 shows that the radial stress resultant $t_{1}$ is a monotonically increasing function of $r$. The circumferential stress resultant $t_{2}$ can have either one or two extreme values.

Acknowledgment. The author would like to thank the Computer Center of the University of Illinois at Chicago Circle for having donated computer time to get the numerical results. 


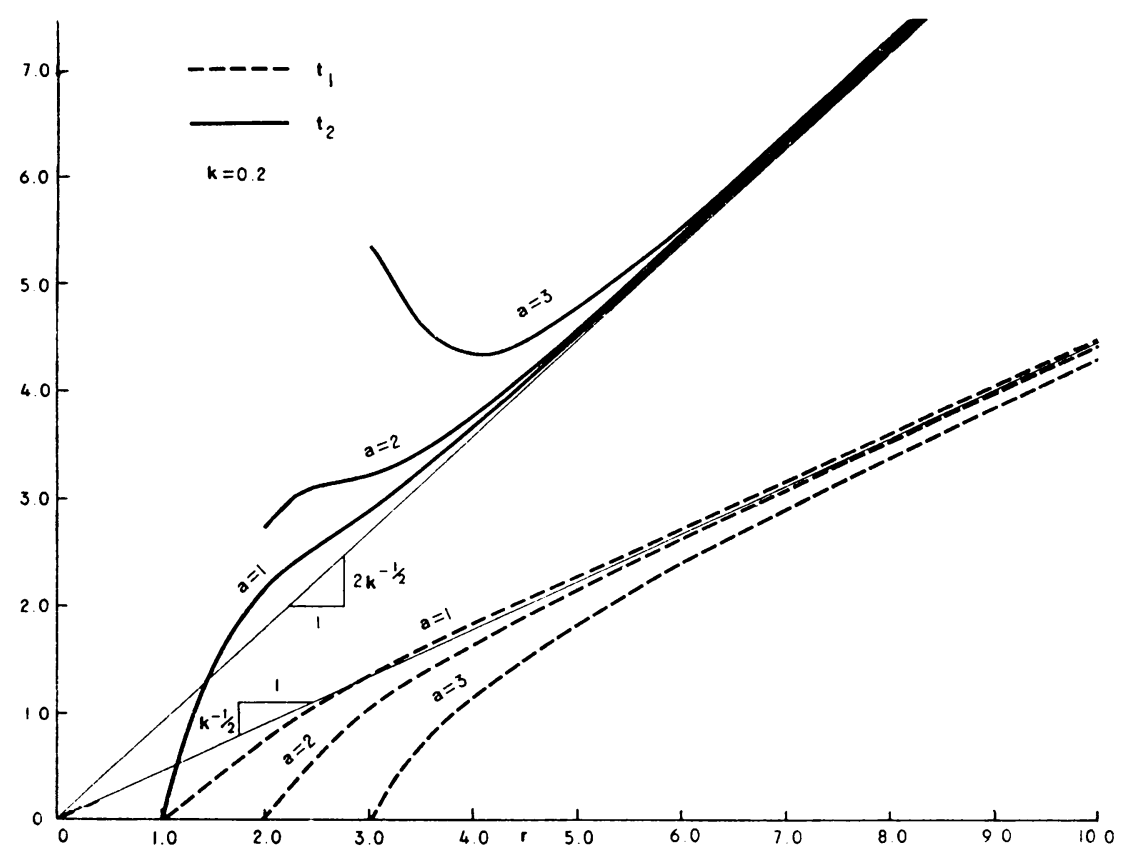

Fig. 5. Stress resultants for a Mooney tube.

\section{REFERENCES}

1. M. Mooney, J. Appl. Phys. 11, 582 (1940)

2. R. S. Rivlin, Philos. Trans. Roy. Soc. London. A241, 379 (1948)

3. A. E. Green and R. T. Shield, Proc. Roy. Soc. London. A202, 407 (1950)

4. R. S. Rivlin and A. G. Thomas, Philos. Trans. Roy. Soc. London. A243, 289 (1951)

5. J. E. Adkins and R. S. Rivlin, Philos. Trans. Roy. Soc. London. A244, 505 (1952)

6. A. E. Green and W. Zerna, Theoretical elasticity, Clarendon Press, Oxford 1954

7. A. E. Green and J. E. Adkins, Large elastic deformation, Clarendon Press, Oxford 1960

8. W. H. Yang, J. Appl. Mech. 4, 942 (1967)

9. H. O. Foster, Int. J. Engrg. Sci. 5, 95 (1967) 